\title{
Quark orbital dynamics in the nucleon - from Ji to Jaffe-Manohar orbital angular momentum
}

\author{
Michael Engelhardt* \\ Department of Physics, New Mexico State University, Las Cruces, NM 88003, USA \\ E-mail: engel@nmsu. edu
}

\begin{abstract}
Quark orbital angular momentum (OAM) in the nucleon can be evaluated directly by constructing the simultaneous distribution of parton transverse position and momentum in a rapidly propagating nucleon, and using it to perform the appropriate average over these parton characteristics. The aforementioned distribution can be accessed via a generalization of the nucleon matrix elements of quark bilocal operators which have been used previously in the lattice evaluation of transverse momentum dependent parton distributions (TMDs). By supplementing these matrix elements with a nonzero momentum transfer, mixed transverse position and momentum information is generated. In the quark bilocal operators, a gauge connection between the quarks must be specified; a staple-shaped gauge link path, as used in TMD calculations, yields Jaffe-Manohar OAM, whereas a straight path yields Ji OAM. A lattice calculation at a pion mass of $518 \mathrm{MeV}$ is presented which demonstrates that the difference between Ji and Jaffe-Manohar OAM can be clearly resolved. The obtained Ji OAM is confronted with the traditional evaluation utilizing Ji's sum rule. Jaffe-Manohar OAM is enhanced in magnitude compared to Ji OAM.
\end{abstract}

34th annual International Symposium on Lattice Field Theory

24-30 July 2016

University of Southampton, UK

${ }^{*}$ Speaker. 


\section{Introduction}

The manner in which the spin of the nucleon is composed of the spins and orbital angular momenta (OAM) of quarks and gluons constitutes an important facet of understanding nucleon structure. The complexity of this question becomes apparent already at the stage of defining appropriate observables; the notion of OAM of quarks and gluons is inherently ambiguous in a gauge theory. Through gauge invariance, quarks are inextricably linked to gluon fields, and any definition of quark OAM will include gluonic effects. Among the many ways in which OAM can be decomposed into quark and gluon contributions, the Jaffe-Manohar [1] and Ji [2] decompositions have garnered the most attention. Within the framework of Lattice QCD, quark OAM has hitherto only been calculated indirectly, via Ji's sum rule [2], which relates total quark angular momentum $J$ to generalized parton distributions. Subtracting quark spin $S$ yields specifically Ji quark OAM, $L=J-S$. On the other hand, Jaffe-Manohar quark OAM has been inaccessible in Lattice QCD using existing methods.

The present work constitutes a first exploration of a method to evaluate quark OAM directly, from simultaneous information about partonic transverse position and momentum in a rapidly propagating nucleon. This information is encoded in generalized transverse momentum-dependent parton distributions (GTMDs) [3-5]. Compared to standard TMDs, which parametrize forward matrix elements of an appropriate bilocal quark operator, GTMDs include, in addition, a momentum transfer. The latter is Fourier conjugate to the quark impact parameter and thus supplements the transverse momentum information with transverse position information. In contrast to Ji's sum rule, this formulation yields access to both Ji as well as Jaffe-Manohar quark OAM, via varying gauge link paths in the aforementioned quark bilocal operator. Indeed, the data to be presented below will continuously and gauge-invariantly interpolate between the two definitions. In view of the similarity between standard TMDs and GTMDs, this work can build to a large extent on the developments made in previous lattice TMD studies $[6,7]$.

\section{Quark orbital angular momentum}

The quark OAM component $L_{3}^{U}$ in a longitudinally polarized nucleon propagating in the 3direction can be accessed via a GTMD matrix element [4],

$$
L_{3}^{U}=\left.\frac{1}{2 P^{+}} \varepsilon_{i j} \frac{\partial}{\partial z_{T, i}} \frac{\partial}{\partial \Delta_{T, j}} \frac{\left\langle p^{\prime}, S^{\prime}=\vec{e}_{3}\left|\bar{\psi}(-z / 2) \gamma^{+} U \psi(z / 2)\right| p, S=\vec{e}_{3}\right\rangle}{\mathscr{S}[U]}\right|_{z^{+}=z^{-}=0, \Delta_{T}=0, z_{T} \rightarrow 0}
$$

A number of remarks are in order concerning this expression. The initial and final nucleon momenta are treated symmetrically, $p=P-\Delta_{T} / 2, p^{\prime}=P+\Delta_{T} / 2$, where the spatial component of $P$ is in 3-direction and the momentum transfer $\Delta_{T}$ is transverse. Since $\Delta_{T}$ is Fourier conjugate to the quark impact parameter $b_{T}$, evaluating the $\Delta_{T}$-derivative at $\Delta_{T}=0$ amounts to averaging $b_{T}$. On the other hand, the transverse quark operator separation $z_{T}$ is Fourier conjugate to the transverse quark momentum $k_{T}$; therefore, evaluating the $z_{T}$-derivative at $z_{T}=0$ amounts to averaging $k_{T}$. Here, the limit $z_{T} \rightarrow 0$ must be taken carefully, since it is associated with ultraviolet divergences. In aggregate, thus, (2.1) yields the average $b_{T} \times k_{T}$, i.e., OAM in the 3-direction. Also the longitudinal quark momentum components are integrated over in view of the specification $z^{+}=z^{-}=0$. 
In the thus constructed average, quark spin direction is immaterial owing to the use of the Dirac structure $\gamma^{+}$. Finally, (2.1) depends on the gauge link $U$ connecting the quark operators, along with a soft factor $\mathscr{S}[U]$ which absorbs divergences associated with the quantum fluctuations of $U$; for present purposes, one may consider $\mathscr{S}[U]$ to include also renormalization factors associated with the quark field operators. This soft factor is the same as for the standard TMD matrix element [8], since (2.1) only differs from the latter in the external state, not the operator. The multiplicative factor $\mathscr{S}[U]$ will be canceled by forming an appropriate ratio below and thus does not need to be specified in more detail. It is in the path of $U$ that different definitions of quark OAM are encoded; (2.1) is a functional of $U$. In the present work, staple-shaped $U \equiv U[-z / 2, \eta v-z / 2, \eta v+z / 2, z / 2]$ are considered, where the arguments of $U$ are positions joined by straight Wilson lines. Thus, the vector $v$ gives the direction of the staple, and the length of the staple is scaled by the parameter $\eta$. For $\eta=0$, one has a straight Wilson line directly connecting the quark operators.

The $\eta=0$ straight gauge link limit corresponds to Ji OAM [9], whereas the $\eta \rightarrow \pm \infty$ limit of a staple extending to infinity yields Jaffe-Manohar OAM [10]. Such a staple link incorporates final state interactions, e.g., in semi-inclusive deep inelastic scattering (SIDIS) processes, with the staple legs corresponding to the direction of propagation of the struck quark. Thus, Jaffe-Manohar quark OAM differs from Ji quark OAM in that it includes the integrated torque accumulated by the struck quark as it leaves the nucleon [11]. In a Lattice QCD calculation, $\eta$ can be varied quasicontinuously, with the Jaffe-Manohar limit achieved by extrapolation. This yields a gauge-invariant interpolation between the Ji and Jaffe-Manohar cases.

In addition, the direction $v$ of the staple needs to be specified. The most straightforward choice for the direction of propagation of the struck quark in a hard scattering process would initially appear to be a lightlike vector. However, such a choice leads to severe rapidity divergences, which are regulated in the scheme advanced in $[12,13]$ by taking $v$ off the light cone into the spacelike region. The matrix element (2.1) determining quark OAM therefore depends on the additional Collins-Soper type parameter

$$
\hat{\zeta}=\frac{v \cdot P}{\sqrt{\left|v^{2}\right|} \sqrt{P^{2}}}
$$

The light-cone limit corresponds to $\hat{\zeta} \rightarrow \infty$.

As in lattice TMD studies [6,7], an appropriate ratio of quantities can be employed to cancel the soft factor $\mathscr{S}[U]$. A suitable quantity for this purpose is the number of valence quarks

$$
n=\left.\frac{1}{2 P^{+}} \frac{\left\langle p^{\prime}, S^{\prime}=\vec{e}_{3}\left|\bar{\psi}(-z / 2) \gamma^{+} U \psi(z / 2)\right| p, S=\vec{e}_{3}\right\rangle}{\mathscr{S}[U]}\right|_{z^{+}=z^{-}=0, \Delta_{T}=0, z_{T} \rightarrow 0}
$$

which only differs from (2.1) by omitting the weighting with $b_{T} \times k_{T}$ (in terms of the Fourier conjugate variables), and thus counts quarks. The soft factor $\mathscr{S}[U]$ is even in $z_{T}$, and thus cancels when forming the ratio $L_{3}^{U} / n$. Furthermore, at finite lattice spacing $a$, the derivative with respect to $z_{T}$ in (2.1) is realized as a finite difference, leading to the renormalized quantity evaluated in practice,

$$
\frac{L_{3}^{U}}{n}=\left.\frac{1}{d a} \varepsilon_{i j} \frac{\frac{\partial}{\partial \Delta_{T, j}}\left(\Phi\left(d a \vec{e}_{i}\right)-\Phi\left(-d a \vec{e}_{i}\right)\right)}{\Phi\left(d a \vec{e}_{i}\right)+\Phi\left(-d a \vec{e}_{i}\right)}\right|_{z^{+}=z^{-}=0, \Delta_{T}=0}
$$


where summation over the transverse indices $i$ and $j$ is implied, and the abbreviation $\Phi\left(z_{T}\right)=$ $\left\langle p^{\prime}, S^{\prime}=\vec{e}_{3}\left|\bar{\psi}(-z / 2) \gamma^{+} U \psi(z / 2)\right| p, S=\vec{e}_{3}\right\rangle$ has been introduced. The aforementioned finite difference is evaluated over a fixed number of lattice spacings $d$, approximating the derivative with respect to $z_{T}$ as $a \rightarrow 0$. The stability of (2.4) with varying $d$ will be examined in the discussion of numerical results below.

\section{Lattice calculation and results}

To perform a lattice calculation of the ratio (2.4), the problem must be boosted into a Lorentz frame in which the TMD operator entering $\Phi\left(z_{T}\right)$ exists at a single time. There is no obstacle to this, given that the directions of $z$ and $v$ are both spacelike, cf. the discussion above in connection with eq. (2.2). In the frame preferred for the lattice calculation, $v$ points in the longitudinal 3direction, whereas $z_{T}$ is transverse, in the direction orthogonal to the momentum transfer $\Delta_{T}$. In this frame, $\Phi\left(z_{T}\right)$ can be evaluated using standard Lattice QCD methods. Numerical data for the ratio (2.4) were obtained in a mixed action scheme employing domain wall valence quarks on a

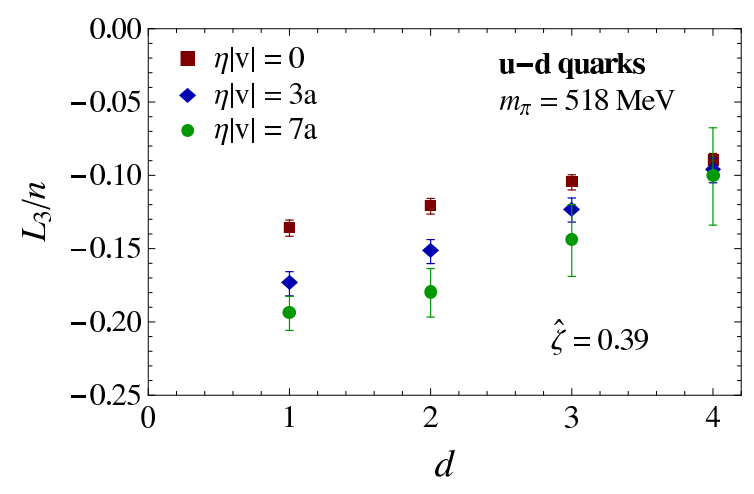

Figure 1: Quark OAM as a function of the number of lattice spacings $d$ used to construct the derivative with respect to $z_{T}$ in (2.4).

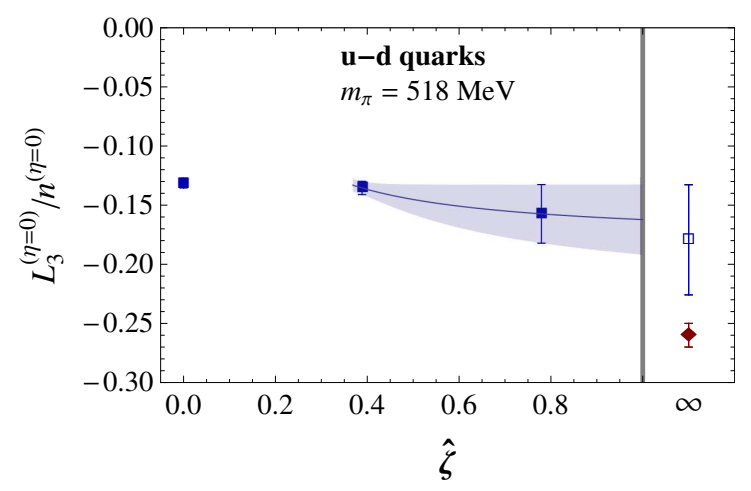

Figure 2: Ji OAM as a function of $\hat{\zeta}$, with an ad hoc extrapolation to infinite $\hat{\zeta}$ (open square). The filled diamond represents the value extracted on the same ensemble via Ji's sum rule.
MILC 2+1-flavor gauge ensemble [14] constituted of $20^{3} \times 64$ lattices with a spacing of $a=0.12 \mathrm{fm}$ and a pion mass $m_{\pi}=$ $518 \mathrm{MeV}$. The source-sink separation employed was $9 a=1.07 \mathrm{fm}$. The longitudinal nucleon momentum components $P_{3}=$ $0,2 \pi /(a L), 4 \pi /(a L)$ were included in the calculation, where $L=20$ denotes the spatial lattice extent. This corresponds to CollinsSoper parameters $\hat{\zeta}=0,0.39,0.78$. These values are appreciably below the region in which perturbative evolution in $\hat{\zeta}$ applies; in view of this, merely an ad hoc extrapolation to large $\hat{\zeta}$ will be contemplated below. The most severe limitation of the gathered data set, however, lies in the momentum transfer $\Delta_{T}$. Eq. (2.4) calls for the evaluation of the derivative with respect to $\Delta_{T}$ at $\Delta_{T}=0$; this derivative was estimated by a finite difference using the lowest nonzero $\Delta_{T}$ available, which, using standard periodic boundary conditions, is $\Delta_{T}=4 \pi /(a L)$ in view of the symmetric treatment of the initial and final nucleon momenta, $p=P-\Delta_{T} / 2, p^{\prime}=$ $P+\Delta_{T} / 2$. This amounts to a substantial momentum transfer, and forming a finite difference using data at this value as opposed to values very close to zero is expected to lead 

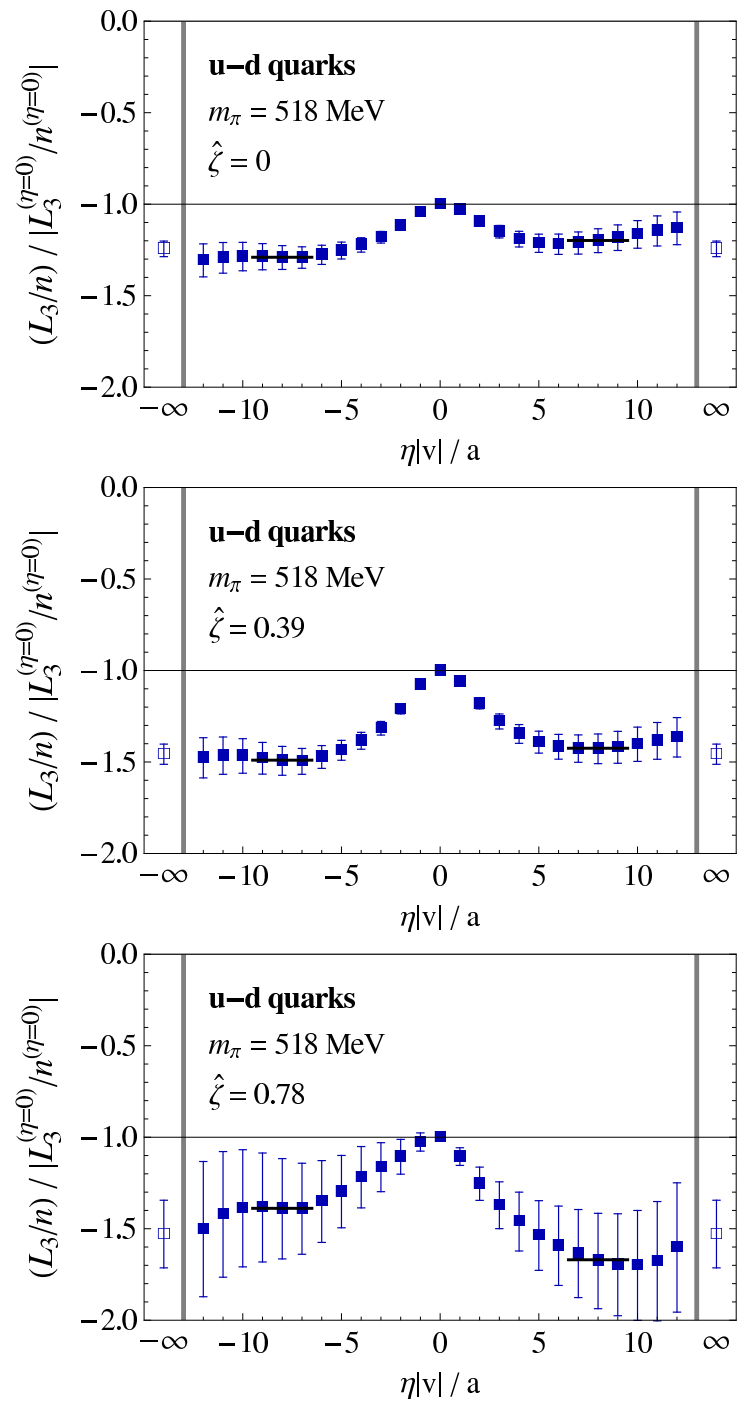

Figure 3: Quark OAM as a function of staple length $\eta$, normalized to the modulus of the $\eta=0 \mathrm{Ji}$ OAM value. Asymptotic values were extracted by averaging over data at $\eta|v| / a= \pm 7, \pm 8, \pm 9$. to a substantial underestimate of the $\Delta_{T}-$ derivative, in view of the typical decay of form factors with the momentum transfer.

Turning to the numerical data, which, to be definite regarding flavor content, will be shown for the proton, Fig. 1 displays data for $L_{3}^{U} / n$ as a function of the number of lattice spacings $d$ used to construct the $z_{T^{-}}$ derivative, for several staple lengths $\eta$ at a fixed $\hat{\zeta}=0.39$. The ratio is quite stable under changes of $d$, with the variation decreasing towards smaller values of $d$, despite the, in principle, singular nature of the $z_{T} \rightarrow 0$ limit. While a more thorough investigation of this limit will require calculations at several lattice spacings, Fig. 1 supports the notion that quark OAM can be estimated via the ratio (2.4) with fairly little ambiguity using small values of $d$. All data presented in the following were obtained using $d=1$.

Focusing, to begin with, on the $\eta=0$ Ji limit, Fig. 2 shows results for Ji quark OAM at the three available values of $\hat{\zeta}$, together with an extrapolation using the ad hoc fit ansatz $A+B / \hat{\zeta}$. This ansatz proved to fit data for the Boer-Mulders TMD ratio well in [7]. Also displayed for comparison is the value for Ji quark OAM extracted on the same gauge ensemble using the standard method utilizing Ji's sum rule [15]. The data gathered here underestimate the $\mathrm{Ji}$ sum rule value, a bias that is not unexpected in view of the poor approximation of the

$\Delta_{T}$-derivative, as discussed above. It seems plausible that an accurate evaluation of this derivative will bring the values obtained with the two methods to match. In the following, data will be shown relative to the $\eta=0 \mathrm{Ji}$ value in order to roughly cancel this systematic bias.

Fig. 3 displays quark OAM data as a function of the staple length for the available values of $\hat{\zeta}$, exhibiting the transition from Ji to Jaffe-Manohar OAM. Starting with Ji quark OAM at $\eta=0$, the struck quark in a deep inelastic scattering process accumulates torque as it is leaving the nucleon, to finally end up with Jaffe-Manohar OAM at large $\eta$. The effect is substantial, can be clearly resolved in the data, and is directed such as to enhance the magnitude of OAM compared to the $\eta=0$ value. It increases with $\hat{\zeta}$, and thus is likely to survive the extrapolation to large $\hat{\zeta}$. Fig. 4 displays such an extrapolation for the integrated torque $\tau_{3}=L_{3}^{(\eta=\infty)} / n^{(\eta=\infty)}-L_{3}^{(\eta=0)} / n^{(\eta=0)}$ alone, 


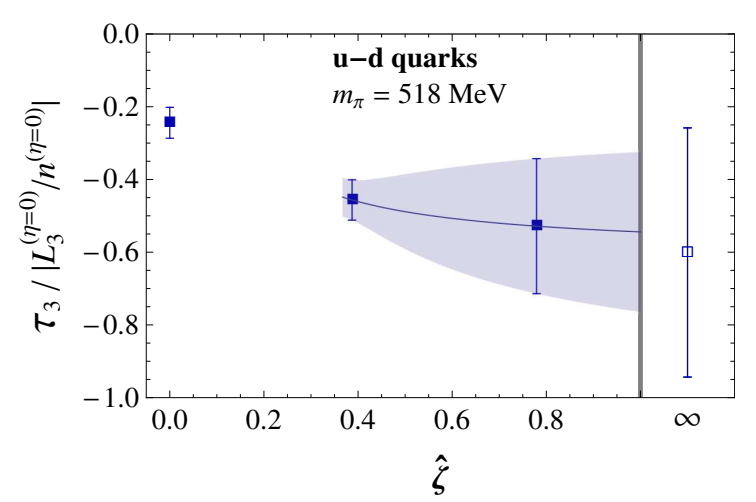

Figure 4: Torque accumulated by the struck quark, cf. main text, normalized to the modulus of the $\eta=0 \mathrm{Ji}$ OAM value. using again the fit ansatz $A+B / \hat{\zeta}$. The extrapolated integrated torque is roughly one half of the originally present Ji quark OAM.

All data shown up to this point have been for the isovector $u-d$ quark combination, in which disconnected contributions to $\Phi\left(z_{T}\right)$ exactly cancel. Fig. 5 shows flavor-separated data at $\hat{\zeta}=0.39$, along with the isoscalar $u+d$ total quark OAM, analogous to Fig. 3 . In these data, disconnected contributions are omitted; at the fairly high pion mass $m_{\pi}=$ $518 \mathrm{MeV}$ considered in this work, they are not expected to be significant. The flavorseparated data show that the well-known cancellation between $u$ - and $d$-quark OAM [15] persists away from the $\eta=0 \mathrm{Ji}$ limit.

\section{Conclusions and outlook}

The direct evaluation of quark OAM in the nucleon via GTMD-related quantities is feasible and allows one to access not only Ji, but also Jaffe-Manohar OAM. By generalizing the TMD matrix element employed in previous lattice TMD studies $[6,7]$ to non-vanishing momentum transfer, quark transverse momentum information is supplemented with impact parameter information, permitting the extraction of OAM. Cancellation of multiplicative soft factors and quark renormalization constants is achieved by forming an appropriate ratio; ultimately, one evaluates OAM in units of the number of valence quarks. The difference between the Ji and Jaffe-Manohar definitions is encoded in the form of the gauge link connecting the quark fields in the bilocal TMD operator; a

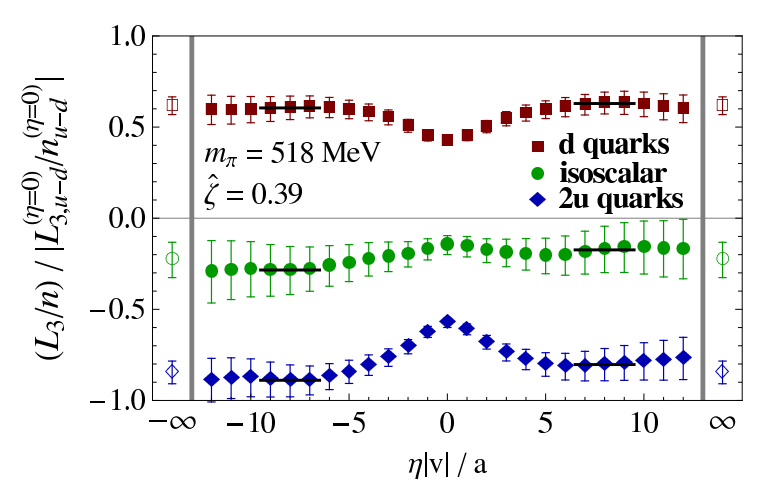

Figure 5: Flavor-separated OAM, analogous to Fig. 3. The $u$-quark data have been multiplied by 2 to compensate for $n=2$ in the $u$-quark case. Isoscalar (total) OAM was obtained by adding the " $d$ " and " $2 u$ " data. Normalization is still by the magnitude of $u-d \mathrm{Ji}$ OAM, i.e., at $\eta=0$, the " $2 u$ " and " $d$ " data differ by unity. straight gauge link yields Ji OAM, whereas an (infinitely long) staple-shaped gauge link yields its Jaffe-Manohar counterpart. In the lattice calculation, a quasi-continuous gauge-invariant interpolation between these two limits is obtained by varying the length of the staple. Physically, this amounts to observing the struck quark in a deep inelastic scattering process start with Ji OAM and then accumulate torque due to final state interactions until it asymptotically attains Jaffe-Manohar OAM. This effect is seen to be substantial, enhancing the quark's OAM by an increment amounting to roughly one half of the original Ji OAM.

The principal shortcoming of the data set generated in the present work lies in the 
estimate of the derivative with respect to momentum transfer $\Delta_{T}$ in the $\Delta_{T}=0$ limit in (2.4). It was obtained via a finite difference using data at a substantial value of $\Delta_{T}$, which appreciably underestimates the derivative, causing, presumably, the discrepancy observed in Fig. 2. Work is underway to completely remove this systematic bias by employing a method to evaluate the derivative exactly [16]. Furthermore, the treatment of larger values of $\hat{\zeta}$, accessible using larger nucleon momenta $P$, is desirable; the use of improved finite momentum nucleon sources [17] will be helpful in this respect. Also the exploration of quark OAM evolution via studies at varying lattice spacings is of interest, and investigations at lighter pion masses must be pursued.

\section{Acknowledgments}

This work benefited from fruitful discussions with M. Burkardt, W. Detmold, J. Green, S. Liuti, C. Lorcé, S. Meinel, B. Musch, J. Negele, S. Syritsyn and B. Yoon. Computations were performed using resources provided by the U.S. DOE Office of Science through facilities of the USQCD collaboration at Jefferson Lab, employing the Chroma software suite [18]. The MILC collaboration is acknowledged for providing the gauge ensemble analyzed in this work. This work was supported by the U.S. DOE through grant DE-FG02-96ER40965, and through the TMD Topical Collaboration.

\section{References}

[1] R. Jaffe and A. Manohar, Nucl. Phys. B337, 509 (1990).

[2] X. Ji, Phys. Rev. Lett. 78, 610 (1997).

[3] S. Meißner, A. Metz and M. Schlegel, JHEP 0908, 056 (2009).

[4] C. Lorcé and B. Pasquini, Phys. Rev. D 84, 014015 (2011).

[5] A. Rajan, A. Courtoy, M. Engelhardt and S. Liuti, Phys. Rev. D 94, 034041 (2016).

[6] B. Musch, P. Hägler, M. Engelhardt, J. Negele and A. Schäfer, Phys. Rev. D 85, 094510 (2012).

[7] M. Engelhardt, P. Hägler, B. Musch, J. Negele and A. Schäfer, Phys. Rev. D 93, 054501 (2016).

[8] M. Echevarria, A. Idilbi, K. Kanazawa, C. Lorcé, A. Metz, B. Pasquini and M. Schlegel, Phys. Lett. B759, 336 (2016).

[9] X. Ji, X. Xiong and F. Yuan, Phys. Rev. Lett. 109, 152005 (2012).

[10] Y. Hatta, Phys. Lett. B708, 186 (2012).

[11] M. Burkardt, Phys. Rev. D 88, 014014 (2013).

[12] S. M. Aybat and T. Rogers, Phys. Rev. D 83, 114042 (2011).

[13] J. C. Collins, Foundations of Perturbative QCD (Cambridge University Press, 2011).

[14] C. Aubin, C. Bernard, C. DeTar, J. Osborn, S. Gottlieb, E. Gregory, D. Toussaint, U. Heller, J. Hetrick and R. Sugar, Phys. Rev. D 70, 094505 (2004).

[15] J. D. Bratt et al. [LHP Collaboration], Phys. Rev. D 82, 094502 (2010).

[16] G. M. de Divitiis, R. Petronzio and N. Tantalo, Phys. Lett. B718, 589 (2012).

[17] G. Bali, B. Lang, B. Musch and A. Schäfer, Phys. Rev. D 93, 094515 (2016).

[18] R. Edwards and B. Joó [SciDAC Collaboration], Nucl. Phys. Proc. Suppl. 140, 832 (2005). 